The Biological Seiences group, under the chairmanship of Prof. F. W. Sansome, heard two papers on cocoa-one, "Comparable Growth Rates of Amazon and Amelonado Cocoa and Responses to Fertilizer", and the other, "Problems in the Assessment of Cocoa Varieties". The third paper was entitled "Liver Cirrhosis in Pigs in Ghana", in which the speaker, Mr. D. Zwart, introduced for discussion the problem of liver cirrhosis which attacked the local black pigs, and later also a few of the European ones, when kept under semi-intensive conditions.

The final full session day of the conference, April 3, opened with one of the popular topics, "Tuberculosis in Ghana", by the tuberculosis specialist in Ghana, Dr. A. Koch. Then followed a general paper by Prof. McCallien on "Seas and Oceans", treated with reference to geological problems in recent oceano. graphic studies.
Quite related to this topic was the next paper on "Outboard-Motor as a Prime Mover in the Development of Fisheries in Ghana", by Mr. E. A. Kwei, of the Government Fisheries Department. Among the other papers for the day were "The Correlation of Field, Laboratory and Analytical Studies of Engineering Structures", by Prof. Royston-Jones, of the Engineering Department, and "Classification of Black and Grey Clays of Ghana using the New U.S. System" presented by three workers led by Mr. H. B. Obeng, of the Scientific Services.

The closing address to round off the conference was given by Mr. H. O. Walker, former chief meteorologist, who made special mention of the many new Ghanaian scientists.

Arrangements are being made to publish the proceedings of the conference in the new scientific journal of the Association. J. YANNEY WILSON

\title{
SATELLITES AND GEODESY
}

$\mathrm{O}^{\mathrm{N}}$ N March 24, 1961, the Royal Astronomical Society held, at its rooms in Burlington House, a geophysical discussion on "Technique of Optical Observations". The chairman for the meeting was Dr. A. H. Cook (National Physical Laboratory), who opened by remarking that the exploration of the major feature of the Earth's gravitational potential by the conventional methods of geodesy was a topic which had often been discussed at meetings of the Society. This meeting, however, would be concerned rather with the elucidation of small-scale features and the use of observations of Earth satellite positions for this purpose.

The discussion was opened by short papers from four speakers, of whom the first was Brigadier G. Bomford. Geodesists had no difficulty, he said, in deriving the size, latitude and longitude and an appropriate geoid for a piece of land, using a variety of well-known astronomical measurements. The problem was to relate the resulting co-ordinate systems for two or more land masses which were separated by a wide sea. If there were no gaps of more than, say, 500 miles to be spanned, the land masses might be connected in latitude and longitude by radar, but this would not connect the two geoids.

However, the problem might be solved by a sufficient number of simultaneous observations of the position of a satellite from each of the land masses to be connected. It was possible that nonsimultaneous non-overlapping observations might be used, but this might also assume a knowledge of the geoid. Brigadier Bomford suggested that such observations would need, in order to be useful, to lead to measurements of position with an accuracy of 30 miles in a horizontal and 10 miles in a vertical direction. Connexions of this kind were required, for example, between Europe and America, across the Mediterranean and between many separated land masses in and around the Pacific.

Dr. R. d'E. Atkinson (Nautical Almanack Office) discussed the astrometric techniques which might be employed, of which there were several. One of the most convenient required the satellite to emit bright, short-duration flashes of light, which would then be photographed against the stars using a camera with an equatorial drive. If the stations were at known longitudes and latitudes, then the time of the flash was in general determined, but if longitude errors in the positions were to be taken out, a timing system with an accuracy of $1 \mathrm{msec}$. was required.

In this way, accuracies in the star positions of $0.05^{\prime \prime}$ could be obtained, but this was because the long exposure reduced errors due to 'seeing' (that is, irregular variations in tropospheric refraction). At low altitudes this effect might be very important and unfortunately connexions over long distances would usually involve large zenith distances. Thus, at a zenith distance of $70^{\circ}$ in a particular case, he had found that 'seeing' increased the angular diameter of the image from $2^{\prime \prime}$ to $4 \cdot 8^{\prime \prime}$, suggesting that the probable error of a single observation of a satellite might be $2^{\prime \prime}$, equivalent to perhaps \pm 60 miles in position. But with multiple observations there would be an improvement.

Alternatively, the conventional method of observing a satellite by reflected sunlight could be used, the observations being made by means of a camera having a shutter which would make timed breaks in the trail. This would be nearly as accurate. The principal problem in this case was to obtain east-west connexions over long distances, as each of the stations could not then be in the dark.

The third paper was read by Dr. A. P. Willmore (University College, London), who pointed out that a principal instrumental difficulty in making optical observations of satellite positions arose from the fact that, viewed from a point on the Earth's surface, a satellite moves with an angular velocity which is neither constant nor accurately predictable. The problem was then to obtain a sufficient exposure to record its position. The sensitivity of a detecting system might in general be limited in any of three ways: by the necessity for recording at least a few photon events in each image element, by statistical fluctuations in the background due to the night sky or by system noise (such as chemical fog, in the case of a photographic emulsion). When these were examined for any but a rather small optical system, it was found that at the high quantum yields characteristic of a photoelectric detector, the limitation always arose from the night sky background, while at the lower quantum yield of the photographic 
plate, chemical fog determined the sensitivity. It could be said that the practical limits for stationary detectors were at about mag. 9 for a photographic and mag. 11 for a photoelectric detector. It appeared that by tracking the camera at the predicted angular rate of the satellite, a gain of about mag. 5 could be obtained.

In fact, the only precise tracking system of high sensitivity in operation was photographic. This was operated by the Smithsonian Astrophysical Observatory, using the specially designed Baker-Nunn cameras. These have an $f / 1$ Super-Schmidt optical system of 20 in. focal length. The cameras are tracked at the predicted angular rate and satellites at a brightness of mag. 13 have been recorded.

However, systems using photoelectric detectors are being studied actively-one, for example, using image intensification by Prof. J. D. Magee at the Imperial College of Science and Technology, London, and another, using photomultipliers, at University College, London.

The advertised speaker for the last paper, $\mathrm{Mr}$. W. H. Guier (Johns Hopkins University), was unfortunately unable to appear, and his place was taken, at rather short notice, by Dr. G. V. Groves (University College, London). This was an account of a radio system which offered some promise as an alternative to optical observations, namely, that employed in the Transit series of satellites. This requires on the satellite two or more high-frequency transmitters, with a very-high-frequency stability over the observing period of several minutes. The frequency of the transmission received at the ground is measured as a function of time. Naturally, it is not constant, but diminishes during the satellite passage, on account of the Doppler shift. The principle of the method can be very roughly described by saying that the total change of frequency during the passage is proportional to the velocity of the satellite, while the maximum rate of change depends both on the velocity and the slant range at closest approach, which can then be determined. It is necessary to make a correction for ionospheric effects, which amount to about 0.2 per cent at $100 \mathrm{Mc}$./s. They are almost inversely proportional to frequency, and can be calculated if two or more transmitters are used.

The method was first applied with encouraging success to the determination of orbital elements of the first satellites by W. H. Guier et al. Later, the transit system was tested in a trajectory determina. tion of an Atlas rocket with an apogee height of 700 $\mathrm{km}$. where comparison with a trajectory determined by radar showed differences of a few $\mathrm{km}$., to which the errors of the radar would have contributed.

It has now also been used on the Transit 1 satellite. Observations were made at six ground stations in the northern hemisphere, and the orbital elements determined, taking the observations for one day at a time, by reproducing to the best fit the observed frequency-time curves. It was found possible to obtain the coefficient of the third harmonic in the gravitational potential with an accuracy of about 5 per cent, and since this produces oscillations of about $5 \mathrm{~km}$. amplitude, it was concluded that the accuracy of measurement was $0.5 \mathrm{~km}$. It seems possible that an improvement in accuracy of an order of magnitude should be obtainable, resulting in position measurement with an accuracy of about 50 miles.

It is intended to use the Transit satellites for navigation, by storing up-to-date orbital elements on a tape recorder in the satellite. These would be read out on command by the ship and combined with Doppler shift observations to obtain the ship's position. During the ensuing discussion, the importance of this for gravity surveys at sea was mentioned by B. C. Browne (Cambridge), as in such observations a principal difficulty is the determination, with adequate precision, of the ship's position.

A. P. WILLMORE

\section{GEOCHEMICAL EFFECTS OF WEATHERING}

\begin{abstract}
A JOINT meeting of the Clay Minerals Group of the Mineralogical Society and the British Society of Soil Science, held in the School of Agriculture, University of Cambridge, during April 6-7, was attended by approximately 100 delegates from home and overseas. At the two paper-reading sessions, the chair was occupied on the afternoon of April 6 by Prof. J. White (Sheffield) and on the morning of April 7 by Dr. H. Greene (Rothamsted Experimental Station). The topic, weathering, was discussed from many points of view (for example, mineralogical, geological, pedological and geochemical) and the conference concluded with the alternative of an excursion led by Mr. C. A. H. Hodge (Rothamsted Experimental Station) to the Brecklands area or informal visits to laboratories of the University.

Prof. C. W. Correns (University of Göttingen), in the introductory paper, outlined the experimental work done on the decomposition of mineral powders by aqueous solutions. Plastic was used in the apparatus and factors such as particle size, rate of flow, temperature, and $p H$ of solution were shown to be important in determining the nature of experimental reactions with potash-felspar, albite, leucite, muscovite, tremolite, olivine and sideromelane. The results of recent experiments on the decomposition
\end{abstract}

of kaolinite and montmorillonite at $p H \mathbf{H}$ could be of significance in the origin of bauxite. The main findings of the whole study were summarized by Dr. G. Nagelschmidt (Sheffield) to the effect that in open systems and at low temperatures, minerals tend to decompose as ions with formation of amorphous residual crusts, whereas in closed systems and at high temperatures secondary minerals are formed. He also directed attention to the formation in Nature of pseudomorphs of kaolin after felspar, and Prof. Correns commented that in an open system and over a long period this might be possible.

An experimental study of the geochemical altera. tion of acidic and basic igneous rocks under varying hydrodynamic and physiochemical conditions was described by Dr. G. Pedro (Versailles). Special attention was paid to the secondary formation of iron and aluminium oxides and hydroxides. The value of the ratio of $\mathrm{SiO}_{2}:\left(R \mathrm{O}+R_{2} \mathrm{O}\right)$ was found to be greater in the leachates than in the unaltered rocks. Prof. White expressed surprise at this, but Dr. Pedro thought that it might possibly be explained by the high $p H$ in the system. Asked whether trace elements had been determined, Dr. Pedro replied that these results would be available very shortly. After several comments on the altera- 